\title{
GENERALIZATION OF COMMON FIXED POINT THEOREMS FOR WEAKLY COMMUTING MAPS BY ALTERING DISTANCES
}

\author{
${ }^{*}$ K. P. R. SASTRY, ${ }^{* *}$ S. V. R. NAIDU, ${ }^{*}$ G. V. R. BABU AND ${ }^{* *}$ G. A. NAIDU
}

\begin{abstract}
The main purpose of this paper is to obtain conditions for the existence of a unique common fixed point for four selfmaps on a complete metric space by altering distances between the points.
\end{abstract}

\section{Introduction}

Obtaining the existence and uniqueness of fixed points for selfmaps on a metric space by altering distances between the points with the use of a certain control function is an interesting aspect. In this direction, Khan, Swaleh and Sessa [1] established the existence and uniqueness of a fixed point for a single selfmap. Recently, Sastry and Babu [4] proved a fixed point theorem by altering distances between the points for a pair of selfmaps.

Pant [2] established a unique common fixed point theorem for four selfmaps by using the minimal type commutativity, contractive and continuity type conditions as follows.

Two selfmaps $A$ and $S$ of a metric space $(X, d)$ are called compatible if $\lim _{n} d\left(A S x_{n}\right.$, $\left.S A x_{n}\right)=0$ whenever $\left\{x_{n}\right\}$ is a sequence such that $\lim _{n} A x_{n}=\lim _{n} S x_{n}=t$ for some $t$ in $X$.

Two selfmaps $A$ and $S$ of a metric space $(X, d)$ are called $R$-weakly commuting at a point $x$ in $X$ if

$$
d(A S x, S A x) \leq R d(A x, S x) \text { for some } R>0
$$

The maps $A$ and $S$ are called pointwise $R$-weakly commuting on $X$ if given $x$ in $X$ there exists $R>0$ such that

$$
d(A S x, S A x) \leq R d(A x, S x)
$$

Let $A$ and $S$ be selfmappings of a metric space $(X, d)$. We call $A$ and $S$ to be reciprocally continuous in $X$ if

$$
\lim _{n} A S x_{n}=A t \text { and } \lim _{n} S A x_{n}=S t
$$

whenever $\left\{x_{n}\right\}$ is a sequence in $X$ such that

$$
\lim _{n} A x_{n}=\lim _{n} S x_{n}=t \text { for some } t \text { in } X .
$$

Received October 20, 1999; revised February 18, 2000.

2000 Mathematics Subject Classification. 47H10.

Key words and phrases. Complete metric space, selfmaps, compatible maps, weakly commuting maps and common fixed points. 
Pant [2] proved the following theorem.

Theorem 1.1. Let $(A, S)$ and $(B, T)$ be pointwise R-weakly commuting pairs of selfmappings of a complete metric space $(X, d)$ such that

i) $A X \subset T X, B X \subset S X$

ii) $d(A x, B y) \leq h M(x, y), 0 \leq h<1, x, y \in X$ where

$$
M(x, y)=\max \left\{d(S x, T y), d(A x, S x), d(B y, T y), \frac{1}{2}[d(A x, T y)+d(B y, S x)]\right\} .
$$

Suppose that $(A, S)$ or $(B, T)$ is a compatible pair of reciprocally continuous mappings. Then $A, B, S$ and $T$ have a unique common fixed point.

We generalize theorem 1.1 by altering distances between the points (Theorem 2.1), using a certain control function,

$\psi: \mathbb{R}^{+} \rightarrow \mathbb{R}^{+}$which is continuous at zero, monotonically increasing, $\psi(2 t) \leq 2 \psi(t)$ and $\psi(t)=0$ if and only if $t=0$.

We also give two examples (Examples 2.2) to show that $\psi$ need not be subadditive.

In the rest of this paper, $(X, d)$ is a complete metric space, $\mathbb{R}^{+}$denotes the nonnegative real line and $\mathbb{Z}^{+}$non-negative integers.

Definition 1.2. Two selfmaps $A$ and $S$ of a metric space $(X, d)$ are called weakly commuting if $d(A S x, S A x) \leq d(A x, S x)$ for every $x$ in $X$. This condition implies that $A S x=S A x$ whenever $A x=S x$.

Notation 1.3. If $A, B, S$ and $T$ are four selfmaps of $(X, d)$ and $\psi$ is as in (1.1.1), we write

$$
\begin{gathered}
M_{\psi}(x, y)=\max \{\psi(d(S x, T y)), \psi(d(A x, S x)), \psi(d(B y, T y)), \\
\left.\frac{1}{2}[\psi(d(A x, T y)]+\psi(d(B y, S x))]\right\} .
\end{gathered}
$$

Definition 1.4. Two selfmaps $A$ and $S$ of a metric space $(X, d)$ are called $\psi$-compatible if $\lim _{n} \psi\left(d\left(A S x_{n}, S A x_{n}\right)\right)=0$ whenever $\left\{x_{n}\right\}$ is a sequence such that $\lim _{n} A x_{n}=\lim _{n} S x_{n}=t$ for some $t$ in $X$.

\section{Main Theorems}

Theorem 2.1. Let $(A, S)$ and $(B, T)$ be weakly commuting pairs of selfmaps of a complete metric space $(X, d)$ and $\psi$ be as in (1.1.1), satisfying

(i) $A X \subset T X, B X \subset S X$ and

(ii) there exists $h$ in $[0,1)$ such that $\psi(d(A x, B y)) \leq h M_{\psi}(x, y)$ for all $x, y \in X$.

Suppose that $(A, S)$ or $(B, T)$ is a $\psi$-compatible pair of reciprocally continuous mappings. Then $A, B, S$ and $T$ have a unique common fixed point. 
Proof. Let $x_{0}$ be any point in $X$. Let $\left\{x_{n}\right\}$ and $\left\{y_{n}\right\}$ be sequences in $X$. Then by (i) we can define, for $n=0,1,2, \ldots$

$$
\begin{gathered}
y_{2 n}=A x_{2 n}=T x_{2 n+1} \\
y_{2 n+1}=B x_{2 n+1}=S x_{2 n+2}
\end{gathered}
$$

We now show that $\left\{y_{n}\right\}$ is a Cauchy sequence.

From (ii), we have

$$
\begin{aligned}
\psi\left(d\left(y_{2 n}, y_{2 n+1}\right)\right)= & \psi\left(d\left(A x_{2 n}, B x_{2 n+1}\right)\right) \\
\leq & h M_{\psi}\left(x_{2 n}, x_{2 n+1}\right) \\
= & h \max \left\{\psi\left(d\left(S x_{2 n}, T x_{2 n+1}\right)\right), \psi\left(d\left(A x_{2 n}, S x_{2 n}\right)\right)\right. \\
& \left.\psi\left(d\left(B x_{2 n+1}, T x_{2 n+1}\right)\right), \frac{1}{2} \psi\left(d\left(B x_{2 n+1}, S x_{2 n}\right)\right)\right\} \\
= & h \max \left\{\psi\left(d\left(y_{2 n-1}, y_{2 n}\right)\right), \psi\left(d\left(y_{2 n}, y_{2 n-1}\right)\right), \psi\left(d\left(y_{2 n+1}, y_{2 n}\right)\right),\right. \\
& \quad \frac{1}{2}\left[\psi\left(d\left(y_{2 n+1}, y_{2 n-1}\right)\right)\right\} \\
= & h \max \left\{\psi\left(d\left(y_{2 n-1}, y_{2 n}\right)\right), \psi\left(d\left(y_{2 n+1}, y_{2 n}\right)\right),\right. \\
& \quad \frac{1}{2}\left[\psi\left(d\left(y_{2 n+1}, y_{2 n-1}\right)\right)\right\} \\
\leq & h \max \left\{\psi\left(d\left(y_{2 n-1}, y_{2 n}\right)\right), \psi\left(d\left(y_{2 n+1}, y_{2 n}\right)\right),\right. \\
& \quad \frac{1}{2}\left[\psi\left(d\left(y_{2 n+1}, y_{2 n}\right)+d\left(y_{2 n}, y_{2 n-1}\right)\right]\right\} \\
\leq & h \max \left\{\psi\left(d\left(y_{2 n-1}, y_{2 n}\right)\right), \psi\left(d\left(y_{2 n+1}, y_{2 n}\right)\right),\right. \\
& \quad \frac{1}{2}\left(\psi\left(2 \max \left\{d\left(y_{2 n+1}, y_{2 n}\right), d\left(y_{2 n}, y_{2 n-1}\right)\right\}\right]\right\} \\
\leq & h \max \left\{\psi\left(d\left(y_{2 n+1}, y_{2 n}\right)\right), \psi\left(d\left(y_{2 n-1}, y_{2 n}\right)\right),\right. \\
& \left.\quad \psi \max \left\{d\left(y_{2 n+1}, y_{2 n}\right), d\left(y_{2 n}, y_{2 n-1}\right)\right\}\right\} \\
= & h \psi\left(d\left(y_{2 n-1}, y_{2 n}\right)\right)
\end{aligned}
$$

In a similar way we can show that

$$
\psi\left(d\left(y_{2 n-1}, y_{2 n}\right)\right) \leq h \psi\left(d\left(y_{2 n-2}, y_{2 n-1}\right)\right)
$$

From (2.1.2) and (2.1.3), we get

$$
\psi\left(d\left(y_{n}, y_{n+1}\right)\right) \leq h^{n} \psi\left(d\left(y_{0}, y_{1}\right)\right)
$$

Also we have, for every positive integer $p$,

$$
\begin{aligned}
\psi\left(d\left(y_{n}, y_{n+p}\right)\right) & \leq \psi\left[d\left(y_{n}, y_{n+1}\right)+d\left(y_{n+1}, y_{n+2}\right)+\cdots+d\left(y_{n+p-1}, y_{n+p}\right)\right] \\
& \leq \psi\left[\left(1+h+\cdots+h^{p-1}\right) h^{n} d\left(y_{0}, y_{1}\right)\right] \\
& \leq \psi\left[\left(\frac{1}{1-h}\right) h^{n} d\left(y_{0}, y_{1}\right)\right]
\end{aligned}
$$


Now for a given $\varepsilon>0$, there exists $N \in \mathbb{Z}^{+}$such that

$$
\psi\left[\left(\frac{1}{1-h}\right) h^{n} d\left(y_{0}, y_{1}\right)\right]<\psi(\varepsilon) \text { for all } n \geq N
$$

This implies $d\left(y_{n}, y_{n+p}\right)<\varepsilon$ for all $n \geq N$.

Hence $\left\{y_{n}\right\}$ is a Cauchy sequence in $X$.

Since $X$ is complete, there is a point $z$ in $X$ such that $y_{n} \rightarrow z$ as $n \rightarrow \infty$. Hence from (2.1.1), we have

$$
\begin{gathered}
y_{2 n}=A x_{2 n}=T x_{2 n+1} \rightarrow z \\
y_{2 n+1}=B x_{2 n+1}=S x_{2 n+2} \rightarrow z
\end{gathered}
$$

Now suppose that $(A, S)$ is a $\psi$-compatible pair of reciprocally continuous mappings. Since $A$ and $S$ are reciprocally continuous, by (2.1.4), we get

$$
A S x_{2 n} \rightarrow A z \text { and } S A x_{2 n} \rightarrow S z
$$

$\psi$-compatibility of $A$ and $S$ imply that

$$
\lim _{n} \psi\left(d\left(A S x_{2 n}, S A x_{2 n}\right)=0\right.
$$

We now show that $A z=S z$. Suppose $A z \neq S z$. Let $\varepsilon=\frac{1}{2} d(A z, S z)$. Then there exists $N \in \mathbb{Z}^{+}$such that

$$
\psi\left(d\left(A S x_{2 n}, S A x_{2 n}\right)\right)<\psi(\varepsilon) \text { for all } n \geq N .
$$

This implies that $d\left(A S x_{2 n}, S A x_{2 n}\right)<\varepsilon$ for all $n \geq N$. Hence by $(2.1 .5), d(A z, S z)<$ $\varepsilon=\frac{1}{2} d(A z, S z)$. a contradiction. Hence

$$
A z=S z
$$

Since $A X \subset T X$, there is a point $w$ in $X$ such that $T w=A z$. By (2.1.6),

$$
T w=A z=S z .
$$

Now, we show that $A z=B w$. Suppose $A z \neq B w$. Now by (ii) we have

$$
\begin{aligned}
\psi(d(A z, B w)) & \leq h M_{\psi}(z, w) \\
& =h \psi(d(B w, T w))=h \psi(d(B w, A z))
\end{aligned}
$$

a contradiction. Hence $A z=B w$. Therefore by (2.1.7),

$$
B w=A z=S z=T w
$$

Since $A$ and $S$ are weakly commuting, we have by (2.1.8), $A S z=S A z$ and

$$
A A z=A S z=S A z=S S z
$$


Since $B$ and $T$ are weakly commuting, we have

$$
B B w=B T w=T B w=T T w
$$

We now show that $A A z=A z$. Suppose $A A z \neq A z$, by (ii), we have

$$
\begin{aligned}
\psi(d(A z, A A z)) & =\psi(d(B w, A A z)) \\
& \leq h M_{\psi}(A z, w) \\
& =h \psi(d(A z, A A z)) \quad(\text { by }(2.1 .8) \text { and }(2.1 .9))
\end{aligned}
$$

a contradiction. Hence $A A z=A z$.

Also, we have, $A A z=S A z$. Therefore $A z$ is a common fixed point for $A$ and $S$. Also suppose $B B w \neq B w$. By (ii), we have

$$
\begin{aligned}
\psi(d(B w, B B w)) & =\psi(d(A z, B B w)) \quad(\text { by }(2.1 .8)) \\
& \leq h M_{\psi}(z, B w) \\
& =h \psi(d(B w, B B w)) \quad(\text { by }(2.1 .8) \text { and }(2.1 .10)) \\
& <\psi(d(B w, B B w))
\end{aligned}
$$

a contradiction. Hence $B B w=B w$ and since $T B w=B B w$, we have $B w$ is a common fixed point for $B$ and $T$. Since $A z=B w$, we have $A z$ is a common fixed point for $A, B$, $S$ and $T$. Uniqueness of a common fixed point follows by (ii). The proof is similar when the pair $(B, T)$ is assumed $\psi$-compatible and reciprocally continuous.

The following two examples show that $\psi$ defined as in (1.1.1) need not be subadditive; consequently $\psi \circ d$ need not be a metric.

\subsection{Examples}

(i) This is an example of a function $\psi: \mathbb{R}^{+} \rightarrow \mathbb{R}^{+}$which is neither continuous nor subadditive but is continuous at zero, monotonically increasing, vanishing only at ' 0 ' and $\psi(2 t) \leq 2 \psi(t)$.

Define

$$
\psi(t)=\left\{\begin{array}{l}
0 \text { if } t=0 \\
2^{-n} \text { if } 2^{-(n+1)} \leq t<2^{-n}, \quad n=0,1,2, \ldots \\
1 \text { if } t \geq 1
\end{array}\right.
$$

Clearly $\psi(2 t) \leq 2 \psi(t)$, but $\psi(s+t) \leq \psi(s)+\psi(t)$ does not hold for $s=0.2$ and $t=0.3$. Clearly $\psi$ is discontinuous at $2^{-n}, n=0,1,2, \ldots$.

(ii) This is an example of a function $\psi: \mathbb{R}^{+} \rightarrow \mathbb{R}^{+}$which is continuous, strictly increasing, vanishing only at ' 0 ' and $\psi(2 t)=2 \psi(t)$ but not subadditive. Let $k$ be a fixed positive real number. 
Define

$$
\psi(t)= \begin{cases}0 & \text { if } t=0 \\ \left(3 t-\frac{1}{2}\right) k & \text { if } \frac{1}{2} \leq t \leq \frac{3}{4} \\ (t+1) k & \text { if } \frac{3}{4}<t<1 \\ 2^{n+1} \psi\left(\frac{t}{2^{n+1}}\right) & \text { if } 2^{n} \leq t<2^{n+1}, n=0,1,2, \ldots \\ \frac{1}{2^{n}} \psi\left(2^{n} t\right) & \text { if } \frac{1}{2^{n+1}} \leq t<\frac{1}{2^{n}}, n=1,2,3, \ldots\end{cases}
$$

Then $\psi\left(\frac{1}{2}+\frac{1}{4}\right)=\psi\left(\frac{3}{4}\right)=\frac{7}{4} k>\frac{3}{2} k=k+\frac{1}{2} k=\psi\left(\frac{1}{2}\right)+\psi\left(\frac{1}{4}\right)$ so that $\psi$ is not subadditive, while $\psi$ has all the other properties mentioned above.

We state the following lemma, which is easy to prove. This lemma is used in the next theorem.

Lemma 2.3. Let $f: \mathbb{R}^{+} \rightarrow \mathbb{R}^{+}$be increasing, continuous at the origin and vanishing only at zero. Then $\left\{t_{n}\right\} \subset \mathbb{R}^{+}$and $f\left(t_{n}\right) \rightarrow 0$ implies $t_{n} \rightarrow 0$.

In the above theorem, we replace reciprocal continuity of $A$ and $S$ by continuity of $S$ and obtain result similar to Theorem 2.1,

Theorem 2.4. Let $(A, S)$ and $(B, T)$ be weakly commuting pairs of selfmaps of a complete metric space $(X, d)$ and $\psi$ be as in (1.1.1) satisfying

(i) $A X \subset T X, B X \subset S X$ and

(ii) there exists $h$ in $[0,1)$ such that

$$
\psi(d(A x, B y)) \leq h M_{\psi}(x, y) \text { for all } x, y \in X .
$$

Suppose that $A$ and $S$ are $\psi$-compatible and $S$ is continuous. Then $A, B, S$ and $T$ have a unique common fixed point.

Proof. Let $x_{0}$ be any point and let $\left\{x_{n}\right\}$ and $\left\{y_{n}\right\}$ be sequences in $X$, define, for $n=0,1,2, \ldots$, by

$$
\begin{aligned}
y_{2 n} & =A x_{2 n}=T x_{2 n+1} \\
y_{2 n+1} & =B x_{2 n+1}=S x_{2 n+2}
\end{aligned}
$$

As in theorem 2.1, the sequence $\left\{y_{n}\right\}$ is a Cauchy sequence in $X$. Since $X$ is complete, there is a point $z$ in $X$ such that

$$
\begin{aligned}
y_{2 n} & =A x_{2 n}=T x_{2 n+1} \rightarrow z \\
\text { and } y_{2 n+1} & =B x_{2 n+1}=S x_{2 n+2} \rightarrow z
\end{aligned}
$$

Since $A$ and $S$ are $\psi$-compatible $A x_{2 n} \rightarrow z$ and $S x_{2 n} \rightarrow z$ implies that

$$
\lim _{n} \psi\left(d\left(S A x_{2 n}, A S x_{2 n}\right)\right)=0
$$

Since $S$ is continuous $S A x_{2 n} \rightarrow S z, S S x_{2 n} \rightarrow S z$ as $n \rightarrow \infty$. Now we show that $\lim _{n} A S x_{2 n}=S z$. By $(2.4 .3)$,

$$
\psi\left(d\left(A S x_{2 n}, S z\right)\right) \leq \psi\left(d\left(A S x_{2 n}, S A x_{2 n}\right)+d\left(S A x_{2 n}, S z\right)\right) \rightarrow 0 \text { as } n \rightarrow \infty .
$$


Hence by above lemma $d\left(A S x_{2 n}, S z\right) \rightarrow 0$ as $n \rightarrow \infty$.

Therefore $\lim _{n} A S x_{2 n}=S z$. Since $A X \subset T X$, for each $n$, there is $w_{2 n}$ in $X$ such that $A S x_{2 n}=T w_{2 n}$. Thus $S S x_{2 n} \rightarrow S z, S A x_{2 n} \rightarrow S z A S x_{2 n} \rightarrow S z$ and $T w_{2 n} \rightarrow S z$ as $n \rightarrow \infty$.

We now show that $\lim _{n} B w_{2 n} \rightarrow S z$. If not, there exists $\varepsilon>0$ and a subsequence $\left\{n_{k}\right\}$ such that $d\left(A S x_{2 n_{k}}, B w_{2 n_{k}}\right)>\varepsilon$ and $\psi\left(d\left(A S x_{2 n_{k}}, S A x_{2 n_{k}}\right)\right)<\varepsilon$ for all $n_{k}$. Therefore

$$
\begin{aligned}
\psi(\varepsilon) \leq & \psi\left(d\left(A S x_{2 n_{k}}, B w_{2 n_{k}}\right)\right) \\
\leq & \left.h M_{\psi}\left(S x_{2 n_{k}}, w_{2 n_{k}}\right)\right) \\
= & h \max \left\{\psi\left(d\left(S S x_{2 n_{k}}, T w_{2 n_{k}}\right)\right), \psi\left(d\left(A S x_{2 n_{k}}, S S x_{2 n_{k}}\right)\right), \psi\left(d\left(B w_{2 n_{k}}, T w_{2 n_{k}}\right)\right),\right. \\
& \left.\frac{1}{2}\left[\psi\left(d\left(A S x_{2 n_{k}}, T w_{2 n_{k}}\right)\right)+\psi\left(d\left(B w_{2 n_{k}}, S S x_{2 n_{K}}\right)\right)\right]\right\} \\
= & h \max \left\{\psi\left(d\left(B w_{2 n_{k}}, T w_{2 n_{k}}\right)\right), \frac{1}{2} \psi\left(d\left(B w_{2 n_{k}}, S S x_{2 n_{k}}\right)\right)\right\} \\
= & h \max \left\{\psi\left(d\left(B w_{2 n_{k}}, A S x_{2 n_{k}}\right)\right), \frac{1}{2} \psi\left(d\left(B w_{2 n_{k}}, S S x_{2 n_{k}}\right)\right)\right\} \\
\leq & h \max \left\{\psi\left(d\left(B w_{2 n_{k}}, A S x_{2 n_{k}}\right)\right), \frac{1}{2} \psi\left(d\left(B w_{2 n_{k}}, A S x_{2 n_{k}}\right)+d\left(A S x_{2 n_{k}}, S S x_{2 n_{k}}\right)\right]\right\} \\
= & h \psi\left(d\left(B w_{2 n_{k}}, A S x_{2 n_{k}}\right)\right)<\psi\left(d\left(B w_{2 n_{k}}, A S x_{2 n_{k}}\right),\right.
\end{aligned}
$$

a contradiction. Hence $\lim _{n} B w_{2 n}=S z$. We now show that $A z=S z$. By (ii) we have

$$
\begin{aligned}
\psi\left(d\left(A z, B w_{2 n}\right)\right) & \left.\leq h M_{\psi}\left(z, w_{2 n}\right)\right) \\
& \left.=h \max \left\{\psi(d(A z, S z)), \frac{1}{2} \psi\left(d\left(A z, T w_{2 n}\right)\right)\right]\right\}
\end{aligned}
$$

Letting $n \rightarrow \infty$, we get

$$
\begin{aligned}
\psi(d(A z, S z)) & \leq h \max \left\{\psi(d(A z, S z)), \frac{1}{2} \psi(d(A z, S z))\right\} \\
& =h \psi(d(A z, S z))
\end{aligned}
$$

a contradiction. Hence $A z=S z$. Since $A X \subset T X$, there exists $w$ in $X$ such that $A z=T w$. Hence $S z=A z=T w$. We now show that $A z=B w$. Suppose $A z \neq B w$, by (ii) we have

$$
\begin{aligned}
\psi(d(A z, B w)) & \leq h M_{\psi}(z, w) \\
& =h \max \left\{\psi(d(B w, T w)), \frac{1}{2} \psi(d(B w, S z))\right\} \\
& =h \max \left\{\psi(d(B w, A z)), \frac{1}{2} \psi(d(B w, A z))\right\} \\
& =h \psi(d(B w, A z))
\end{aligned}
$$

a contradiction. Hence $A z=B w$. Thus $S z=A z=T w=B w$. Since $A$ and $S$ are weakly commuting, we have $A S z=S A z$ and hence $A A z=A S z=S A z=S S z$ and by 
the weakly commuting property of $B$ and $T$, we have $B B w=B T w=T B w=T T w$. The remaining part of the proof is as in Theorem 2.1.

Note. The above theorem is valid if we assume $B$ and $T$ are $\psi$-compatible and $T$ is continuous, instead of similar restrictions on $A$ and $S$.

Note. We observe that the theorem of Pant [2] is a special case of Theorem 2.1 by taking $\psi$ as the identity function.

We conclude the paper with the following open problem:

\section{Open problem.}

Is theorem 2.4 valid if we replace continuity of $S$ by continuity of $A$ or continuity of $T$ by continuity of $B$ ?

\section{References}

[1] M. S. Khan, M. Swaleh and S. Sessa, Fixed point theorem by altering distances between the points, Bull. Austral. Math. Soc., 30(1984), 1-9.

[2] R. P. Pant, Common fixed points of four mappings, Bull. Cal. Math. Soc., 90(1998), 281-286.

[3] R. P. Pant, Common fixed point theorems for contractive mappings, J. Math. Anal. Appl., 226(1998), 251-256.

[4] K. P. R. Sastry and G. V. R. Babu, Some fixed point theorems by altering distances between the points, Ind. J. Pure and Appl. Math., 30(June 1999), 641-647.

*Department of Mathematics, Andhra University, Visakhapatnam-530 003, India.

** Department of Applied Mathematics, Andhra University, Visakhapatnam 530 003, India (Currently at: Dept. of Mathematics, University of Bostswana, Gaborone, Botswana)

*** Department of Mathematics, A. U. Dr. B. R. A. P. G. Gentre, Etcherla, Srikakulam 532 402, India. 